\title{
COMHUMANANITAS
}

Revista ComHumanitas, ISSN: 1390-776X

Vol. 11, núm. 3 (2020), Septiembre - Diciembre 2020

DOI: https://doi.org/10.31207/rch.v11i3.273

\section{Los desafíos éticos de los medios informativos en la cobertura de la Covid-19 1}

\section{The ethical challenges of the news media in the coverage of Covid-19}

\section{Os desafíos éticos da mídia na cobertura da Covid-19}

\author{
Alex Iván Arévalo Salinas² \\ Universidad de Extremadura (España) \\ alexarevalo@unex.es
}

Fecha de recepción: 2 de diciembre de 2020

Fecha de recepción evaluador: 13 de diciembre de 2020

Fecha de recepción corrección: 23 de diciembre de 2020

\begin{abstract}
Resumen
En este artículo se analiza algunos desafíos y cambios que ha impuesto la pandemia del Covid-19 a los medios informativos a través de ejemplos de noticias en el contexto español e iberoamericano. Uno de estos cambios ha sido a restructuración de los sitios web de 10 medios españoles para dar notoriedad a la pandemia. Este escenario implica desafíos en generar contenidos de calidad que contribuyan al entendimiento de este

\footnotetext{
${ }^{1}$ Este artículo es parte del proyecto titulado «Converging Horizons: Production, Mediation, Reception and Effects of Representations of Marginality», código SOC180045 de la convocatoria Anillos de investigación en Ciencias Sociales de la Agencia Nacional de Investigación y Desarrollo de Chile (ANID).

${ }^{2}$ Doctor y Máster en Estudios internacionales de paz, conflictos y desarrollo por la Universitat Jaume I de Castellón, España. Periodista por la Universidad de Playa Ancha. Es profesor e investigador del Departamento de Información y Comunicación de la Universidad de Extremadura, España. ORCID: https://orcid.org/0000-0002-0065-2119.
} 
escenario y evitar la propagación de estereotipos o prejuicios. En este artículo se analiza el caso específico de la representación de los jóvenes durante las limitaciones a la movilidad por los estados de emergencia y alarma. Se evidencia cómo algunos medios informativos, a través de diferentes estrategias, refuerzan la asociación de los jóvenes como infractores de las normativas.

Palabras clave: Medios informativos; Covid-19; periodismo; jóvenes; crisis

\begin{abstract}
This article analyzes some challenges and changes that the Covid-19 pandemic has imposed on the news media through examples of news in the Spanish and Latin American context. One of these changes has been a restructuring of the websites of 10 Spanish media to give visibility to the pandemic. This scenario involves challenges in generating quality content that contributes to understanding this scenario and avoiding the spread of stereotypes or prejudices. This article analyzes the specific case of the representation of young people during mobility limitations. It is evident how some news media, through different strategies, reinforce the association of youth as violators of the regulations.
\end{abstract}

Keywords: Information media; Covid-19; journalism; youth; crisis

\title{
Resumo
}

Este artigo analisa alguns desafios e mudanças que a pandemia de Covid-19 impôs à mídia noticiosa por meio de exemplos noticiosos no contexto espanhol e latinoamericano. Uma dessas mudanças foi a reestruturação dos sites de 10 meios de comunicação espanhóis para dar visibilidade à pandemia. Esse cenário implica desafios na geração de conteúdos de qualidade que contribuam para a compreensão desse cenário e evitem a disseminação de estereótipos ou preconceitos. Este artigo analisa o caso específico da representação de jovens durante limitações de mobilidade devido a estados de emergência e alarme. É evidente como alguns meios de comunicação, por meio de diferentes estratégias, reforçam a associação dos jovens como infratores das normas.

Palavras-chave: Meios de informação; Covid19; jornalismo; jovens garotos; crise

\section{Introducción}

La pandemia del Covid-19 y las restricciones a las interacciones físicas han impuesto cambios en distintos niveles desde la obligatoriedad del teletrabajo, la docencia online o la modificación de las estrategias comerciales, entre otros aspectos. Los medios informativos no han estado exentos de estos cambios, por ejemplo, ante la caída de los ingresos publicitarios, algunas de estas empresas han variado sus modelos de negocio. Dos ejemplos son elpais.com con el cobro por el acceso a sus contenidos, una vez superada las 10 noticias gratuitas, y eldiario.es con la subida del coste de la cuota a sus socios. 
Las catástrofes modifican las dinámicas periodísticas (Domínguez, 2017). Cobra relevancia la información de última hora, las actualizaciones continuas y la lógica del directo en televisión (Wolton, 2002). La diversidad de temas queda en un segundo plano para centralizar la cobertura en el hecho central. A lo que se suma el creciente interés de los ciudadanos por acceder a datos confiables y actualizados que reduzcan la incertidumbre. Según las estadísticas de Google Trends sobre la búsqueda de noticias durante la pandemia, la palabra Coronavirus registró su máxima utilización como criterio de indagación en el periodo del 8 al 14 de marzo de 2020 y para Covid-19 del 9 al 15 de agosto de 2020. El crecimiento comienza para Coronavirus desde el 19 al 25 de enero de 2020 y para Covid-19 del 26 de enero al 1 de febrero de 2020. Como se observa en el gráfico 1, la palabra Coronavirus tuvo una mayor utilización en las indagaciones de los usuarios.

Gráfico 1: Datos sobre la búsqueda de noticias referente a los términos Coronavirus y Covid-19 en un periodo de un año ( 23 de diciembre de 2019 a 22 de diciembre de 2020 )

Interés a lo largo del tiempo Google Trends
coronavirus COVID-19

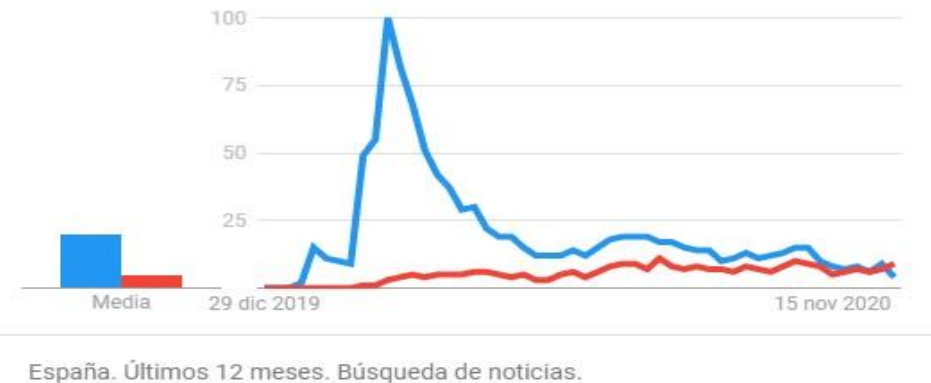

Fuente: Google Trends, fecha de captura de la imagen el 23 de diciembre de 2020

Los medios informativos durante la pandemia han experimentado una serie de desafíos relacionados al resguardo de su calidad periodística y el cumplimiento de su responsabilidad social. En relación a estos aspectos, en este artículo se describen tres desafíos informativos: El primero relacionado con la necesidad de aportar información clara, visible y oportuna. En este sentido, se describen las modificaciones realizadas en las páginas web de 10 medios informativos españoles, 4 diarios impresos (La Razón, El Mundo, ABC y El País) y 6 nativos digitales (eldiario.es, El Confidencial, Okdiario, El Español, Huffpost, El Economista digital), seleccionados por su relevancia informativa. El objetivo es comprobar la visibilidad otorgada al tema de la pandemia en la distribución de su contenido y la utilización de herramientas del periodismo de datos como mapas y gráficos. Los datos son extraídos durante el 5 de abril de 2020.

El segundo desafío analiza los titulares de 286 noticias publicadas por los 10 medios informativos mencionados. El objetivo es cuantificar en sus titulares en qué grado se destacó el número de contagiados en comparación a otros aspectos. Se asume que las cifras de infecciones permiten tener una imagen sobre el ritmo de crecimiento de la 
pandemia y la efectividad de las medidas de contención. De esta manera, se les confiere una mayor relevancia en relación a la cifra de muertos, siendo este último aspecto una consecuencia de los contagios. Los titulares analizados corresponden a todo el mes abril de 2020. En los dos primeros desafíos, se selecciona el mes de abril por ser un espacio temporal en que se establece el estado de alarma en España que obliga a su población a estar confinada en sus domicilios.

Por último, el tercer desafío aborda como caso específico la representación de los jóvenes durante la pandemia a través de ejemplos de noticias publicadas en medios iberoamericanos de Perú, Chile y España. El objetivo es detallar cómo en algunos casos se ha reforzado la tradicional asociación de los jóvenes como grupo infractor. Estas noticias son recopiladas desde los buscadores de los propios medios informativos en el caso de Emol.cl de Chile y El Comercio de Perú y desde Google noticias con las palabras claves jóvenes y Covid-19.

\section{Estado de la cuestión}

En escenarios de alto impacto emocional y físico como atentados terroristas, guerras o crisis sanitarias aumenta la intensidad de las informaciones negativas aplicándose la dinámica de la cobertura en directo, donde se potencia la emoción por sobre el razonamiento (Wolton, 2002) y queda en un segundo plano la contextualización (Barranquero, 2019; Arévalo Salinas, 2020). El discurso emocional y negativo es una vía para atraer la atención de los ciudadanos vinculado al interés estratégico comercial de las empresas de captar más anunciantes. Este contenido instrumental se enmarca en el segundo filtro del modelo de propaganda de Chomsky y Herman (2009) que detalla la influencia que tiene la publicidad en la viabilidad de los proyectos informativos y en las decisiones editoriales. Las estrategias periodísticas privilegian los contenidos que logren captar la atención de las audiencias, dada la relación entre estos indicadores y los niveles de ingreso por publicidad.

Los contenidos negativos como la violencia son coherentes con estos fines porque generan mayores ventas (Arango-Kure, Garz, Rott, 2014). Las informaciones negativas se convierten en una estrategia tentadora por su impacto emocional captando la atención de los ciudadanos. De acuerdo a Taylor (Menéndez, 2014, p. 28), la “información sobre sucesos negativos activa y focaliza más la atención del público, impulsa una mayor reflexión y búsqueda de explicaciones y, en general, tiene un mayor impacto que las noticias de valor positivo". Por su parte, estudios experimentales, que incluyeron el uso de biosensores, evidencian un interés mayor de las personas por las informaciones negativas en comparación a las positivas (Lamberson y Soroka, 2018). Soroka y Adams (2015, p. 15) añaden lo siguiente en relación al papel del periodista sobre este tipo de contenidos: 
News is likely negative in part because news consumers are more attentive to negative information. But the propensity to over-represent negativity in mass media need not be a product of profit-maximization alone. Journalists and editors are also humans, after all, and thus have the same tendencies as their audience.

Por su parte, Casares (2020) reflexiona sobre las percepciones profesionales en torno a las noticias positivas y negativas. Este autor afirma que los periodistas otorgan mayor valor al pesimismo porque hay "una cierta pose intelectual según la cual el pesimismo nos hace más serios, más interesantes o más inteligentes. Afecta en especial a los artículos de opinión" y que las noticias esperanzadoras son consideradas como escasamente críticas.

La relevancia de las informaciones negativas se comprueba en que las malas noticias son uno de los principales criterios de noticiabilidad o valores noticiosos (news value) $)^{i}$ (Menéndez, 2014). De esta forma, "cuantas más negativas sean las consecuencias de un hecho (guerras, delincuencia, violencia, etc.), más probabilidades tiene éste de llegar a convertirse en noticia" (Menéndez, 2014, p. 27-28).

Díaz Rojo (2009) incluye y describe un listado de 19 valores noticiosos, de los cuales elegimos tres por su relación con la pandemia del Covid-19:

5) Drama personal. Es la base de historias o relatos personales cargados de sentimiento que despiertan la sensibilidad del lector por su impacto emocional, como, por ejemplo, las historias dramáticas de enfermos o heridos.

8) Magnitud. Viene determinado por la importancia cuantitativa o cualitativa de un hecho; por ejemplo, el elevado número de afectados por un problema de salud o por una medida política en materia de salud.

10) Negatividad. Son las «malas» noticias, es decir, aquellas que tratan de muertes, tragedias, enfermedades, accidentes, desastres, catástrofes, actos de violencia o agresiones.

Este enfoque negativista, en un escenario como el actual, no contribuye a la estabilidad emocional de las personas como tampoco a la búsqueda de soluciones. La construcción de una exclusiva realidad negativa, funesta y de confrontación, refuerza el miedo y la desmovilización social (Arévalo Salinas, 2020). Es por ello, que se requiere fomentar debates constructivos, contextualizados y de soluciones, con una mayor intensidad de discursos esperanzadores y positivos.

La pandemia ha forzado a los periodistas a trabajar en un escenario con las dificultades propias de las restricciones sanitarias y de aislamiento social, pero también les ha obligado a priorizar la verificación y el contraste de los contenidos, dada la propagación de rumores e informaciones falsas. A lo que suma las exigencias de especialización y de recurrir a fuentes fiables. Al respecto, la Asociación Española de Comunicación científica (2020) recomienda utilizar como fuentes a "personas expertas en disciplinas como medicina preventiva y salud pública, epidemiología, virología, 
inmunología y neumología, entre otras, que pueden ofrecer opiniones y datos rigurosos en las cuestiones relativas a sus especialidades".

Un ejemplo de los potenciales errores que se pueden generar en este contexto, se visualiza en una noticia publicada por el diario El Mundo (2020), en su edición de la Comunidad Valenciana, España, que desmiente un dato difundido por la cadena pública de televisión À punt de la Comunidad Valenciana. En la noticia televisiva, según El Mundo, se menciona la hospitalización de 3 adolescentes y niños en las Unidades de Cuidados Intensivos UCI de la provincia de Castellón. Sin embargo, El Mundo informa de este dato es erróneo a través de la declaración de la Conselleria de Sanidad de la Comunidad Valenciana mencionando que: "sólo se encuentra hospitalizado un paciente menor de edad (13 años) con sospecha de Covid-19 y se encuentra en la UCI, pero su estado es estable", a la vez que se destaca que el Hospital de La Plana "no tiene ningún menor ingresado con patología Covid-19".

Otro desafío periodístico en escenario de crisis es no fomentar estigmatizaciones, prejuicios y estereotipos sobre algunos colectivos. De acuerdo al Ente de Comunicación Nacional en Argentina Enacom (2020), los periodistas durante el Covid-19 deben "evitar que el suministro de noticias conlleve a situaciones de estigmatización o discriminación por nacionalidad, origen ético o social, etario, como de género o de cualquier tipo".

Los jóvenes tradicionalmente han sido representados desde un enfoque criminalizador en el discurso mediático. Se los ha construido como grupo infractor, que no respeta las normas cívicas de convivencia, es decir, como "personas (...) violentas, insolidarias y viciosas" (Asociación Diomira, 2007, p. 309). En términos periodísticos, su cobertura está influenciada por las malas noticias insertados en situaciones de conflictos como la delincuencia y las drogas, entre otros (Menéndez, 2014). Es un discurso generalizador que invisibiliza sus aportaciones sociales. Menéndez (2014) afirma que, en el contexto reciente de España, producto de la crisis económica que experimentó este país desde el cuarto trimestre de 2007, los jóvenes son representados como víctimas por la falta de oportunidades, con noticias que mencionan su situación de desempleo.

A continuación, detallaremos, con algunos ejemplos, los tres desafíos que ha impuesto la emergencia sanitaria en el caso de los medios españoles y latinoamericanos.

\section{Primer desafío: Restructuración de las páginas web para una información de fácil acceso}

Como se ha visto en los datos de Google Trends, en contextos de emergencia crece el interés de los ciudadanos por conocer informaciones actualizadas y de contexto que reduzcan la incertidumbre. Un escenario de la magnitud de la pandemia ha impuesto el desafío de restructurar las páginas web de los medios informativos con el 
objetivo de dar visibilidad a este tema y facilitar el acceso apoyándose en la utilización de herramientas de visualización de datos.

En el caso de los medios españoles, con información extraída el 5 de abril de 2020, se observa la utilización de herramientas de visualización como gráficos y mapas como también la creación de nuevas secciones. De estos 10 medios analizados, 8 aplicaron modificaciones relevantes en su web y en 6 incorporaron herramientas de visualización de datos como los gráficos y mapas de curva. A continuación, se detallan sus diferentes niveles:

En 4 medios informativos (El Mundo, La Razón, El País y Eldiario.es) se crearon recuadros con temas asociados a esta pandemia, en la parte superior de la noticia principal. Por ejemplo, El diario elpais.com lo tituló como "Crisis del coronavirus" compuesto por: un apartado de datos actualizados; la curva actualizada; una guía básica de cómo actuar; una sección podcast y un newsletter. El medio informativo que realizó la restructuración más profunda fue eldiario.es al reemplazar sus secciones tradicionales (Sociedad, Economía, Política) por las vinculadas a esta pandemia (Curva en España, Los síntomas, Así ataca, Historias del coronavirus). Además, eldiario.es insertó un recuadro especial, titulado de la misma forma que el diario elpais.com, compuesto por las siguientes secciones (Lo último, mapas y datos, preguntas y respuestas y newsletter).

Imagen 1. Estructura de eldiario.es durante la pandemia del Covid-19

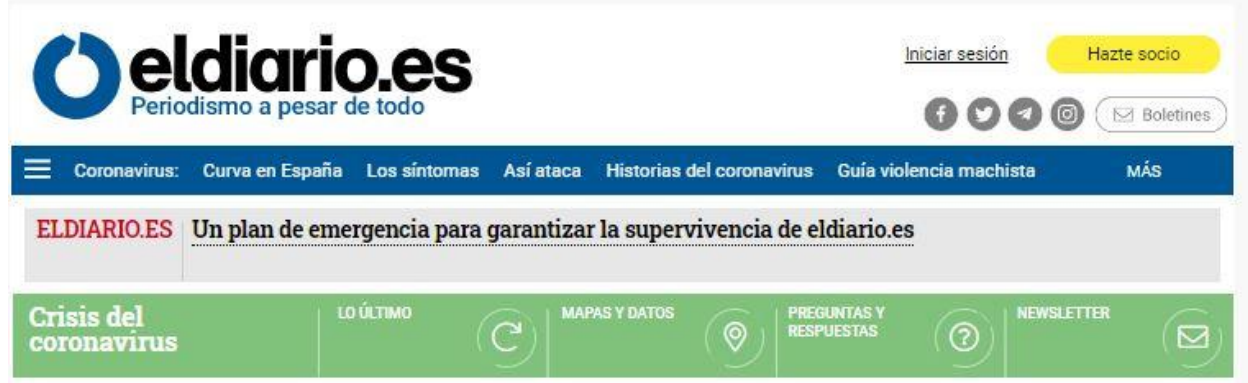

\section{La cifra de fallecidos con coronavirus en las últimas 24 horas desciende a 674 , la menor en 10 días}

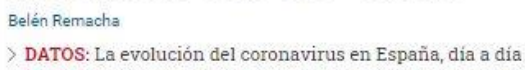

Fuente: eldiario.es, fecha de captura de la imagen el 5 de abril de 2020

En 2 medios informativos (El Confidencial y El Español) la relevancia se centró en potenciar la visibilidad de los mapas de curva ubicándolos arriba la noticia principal. Otros medios incluyeron estas herramientas, pero en lugares secundarios como parte de los recuadros especiales como La Razón, El Mundo, eldiario.es y El País. 


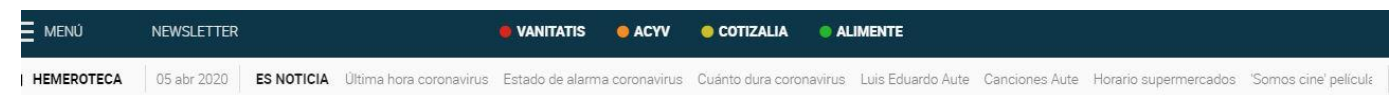

\section{El Confidencial}

ESPAÑA OPINIÓN CULTURA MUNDO TEKNAUTAS DEPORTES TELEVISIÓN VIDEOS PODCASTS OPrevium.

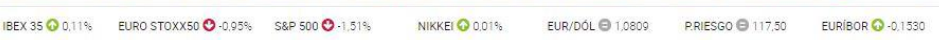

EN PRIMERA PERSONA "Estamos trabajando 16 horas al dia y en la televisión nos insultan"

Crisis Covid-19

350.000

300.000

300.000
250.000

:sando

Fuente: El Confidencial, fecha de captura de la imagen el 5 de abril de 2020

En 2 casos (ABC y Okdiario) se privilegió la visibilidad de datos cuantitativos, incluidos también en recuadros ubicados en la parte superior de la noticia principal. ABC añadió diferentes aspectos numéricos sobre los efectos de la pandemia, tanto a nivel de España como en el mundo, en relación a la cantidad de personas confirmadas con el virus, los muertos en España y los recuperados. En el caso de OKdiario se añadió solamente dos categorías: cantidad de muertos y contagiados.

\section{Imagen 2. Estructura de ABC durante la pandemia del Covid-19}

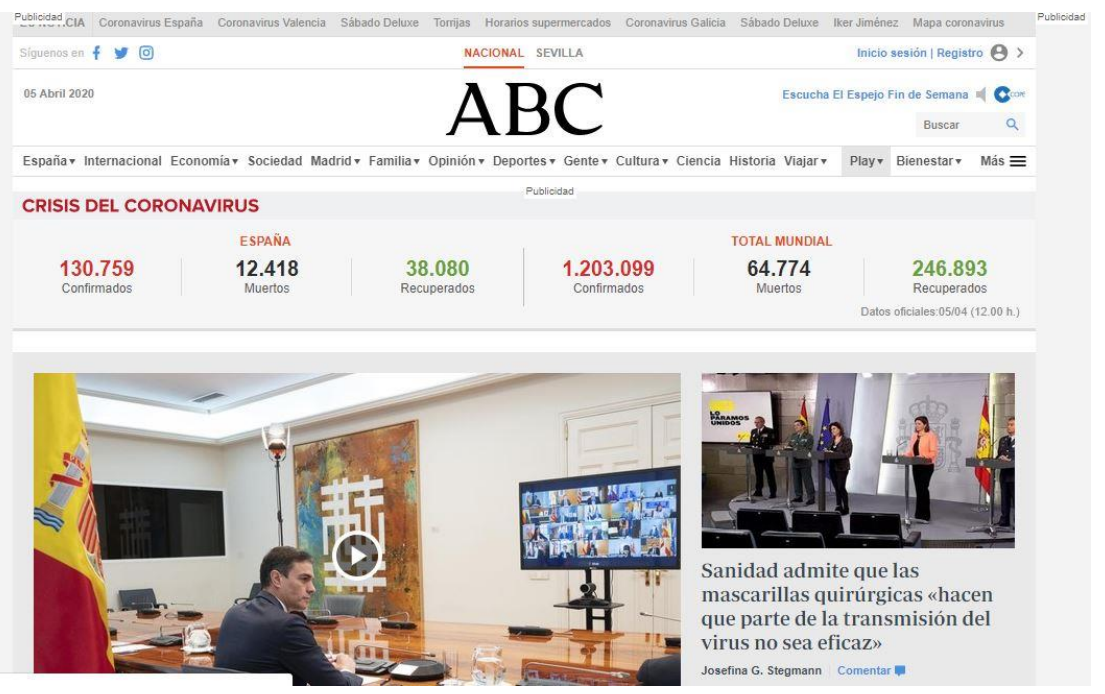

Fuente: ABC, fecha de captura de la imagen el 5 de abril de 2020 
Por último, en Huffpost y El Economista digital la inclusión del tema de la pandemia no supuso modificaciones sustanciales y se creó una sección incorporándolas junto a las tradicionales denominadas Estado de Alarma y Coronavirus, respectivamente.

Imagen 3. Estructura de Huffpost durante la pandemia del Covid-19

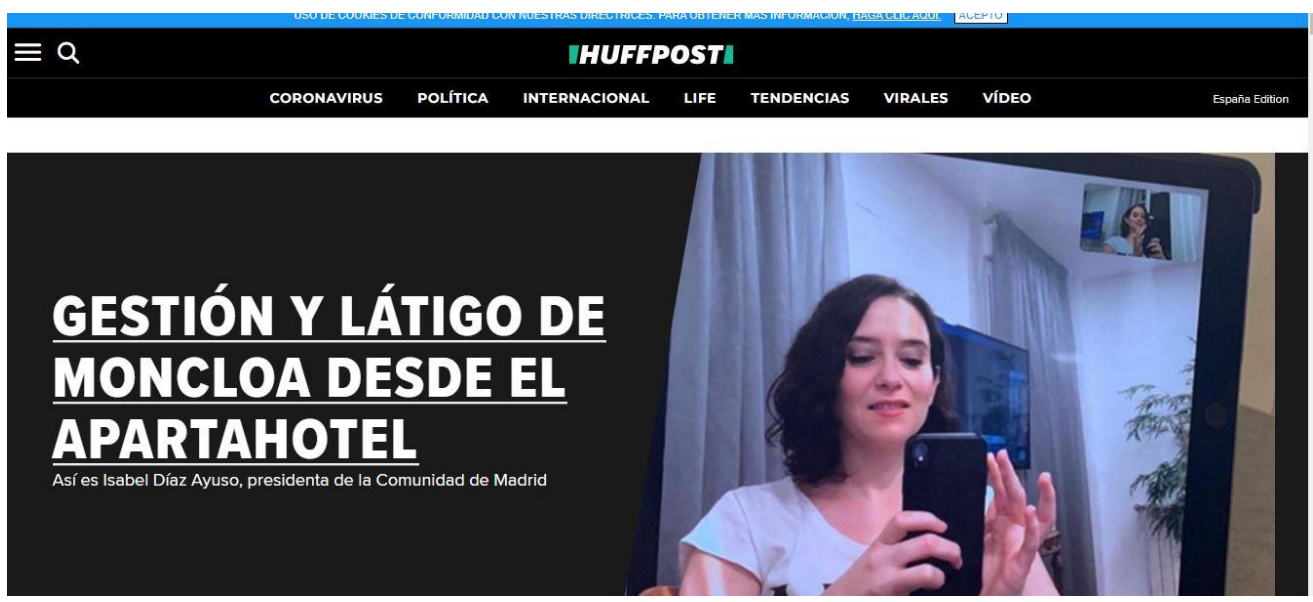

Fuente: Huffpost fecha de captura de la imagen el 5 de abril de 2020

\section{Segundo desafío: El titular y su aporte para el conocimiento de la evolución de la pandemia}

En este segundo desafío cuantificaremos la relevancia otorgada en los titulares al número de contagiados en los 10 medios informativos españoles mencionados en el anterior apartado (La Razón, El Mundo, ABC y El País, eldiario.es, El Confidencial, Okdiario, El Español, Huffpost, El Economista digital). Como se señaló, se asume que las cifras de infecciones permiten tener una imagen sobre el ritmo de crecimiento y la efectividad de las medidas de contención. De esta manera, se les confiere una mayor importancia en relación a la cifra de muertos, siendo este último aspecto una consecuencia de los contagios.

Se revisará la base datos MyNews, un servicio de hemeroteca que incluye 140 periódicos. Se recopilan las noticias que contienen las palabras Muertos, Contagiados y Sanidad durante el mes de abril de 2020 en los 10 medios informativos mencionados. La palabra Sanidad es seleccionada por su relación con el Ministerio de Sanidad, organismo encargado de dar los reportes diarios sobre el impacto de esta pandemia en España. La plataforma MyNews registró 652 resultados, de las cuales 600 se encuentran disponibles para su consulta. Se hace una delimitación para seleccionar aquellos titulares que informan sobre los datos actualizados del impacto de la pandemia e incluyan los siguientes aspectos: número de muertos, número de contagiados, número de recuperados o titulares que combinen varias variables. El corpus final de revisión alcanzó los 286 titulares. Cabe destacar, que se contabilizaron los sinónimos como fallecidos en el caso de las muertes, entre otras. 
Los resultados expresan que la principal forma de titulación combina los datos del número de muertos con otras variables en 146 casos, siendo frecuente la combinación entre los datos de los fallecidos y las personas infectadas por el virus. En segundo lugar, se contabilizan 114 titulares que informan exclusivamente del número de muertos. En tercer lugar, se registran 20 casos con titulares que hacen referencia a la cifra de contagiados. Finalmente, en 6 casos el titular menciona al número de recuperados.

Los datos expresan una preferencia por el número de fallecidos como se comprueba en la elevada diferencia entre los titulares que se centran exclusivamente en los datos de los fallecidos, 146 casos, en comparación a los contagiados con 20 y los recuperados con 6. En el siguiente cuadro se puede visualizar las diferencias entre los distintos medios:

Tabla 1: Preferencias en la forma de titular en noticias que incluyen las palabras claves Muertes, Contagiados y Sanidad

\begin{tabular}{|l|c|c|c|c|}
\hline \multicolumn{1}{|c|}{ Medios } & $\begin{array}{c}\text { Número de } \\
\text { muertos }\end{array}$ & $\begin{array}{c}\text { Número de } \\
\text { contagiados }\end{array}$ & $\begin{array}{c}\text { Número de } \\
\text { recuperados }\end{array}$ & $\begin{array}{c}\text { Combinan } \\
\text { variables }\end{array}$ \\
\hline Abc.es & 38 & 4 & 2 & 61 \\
\hline El Confidencial & 14 & 4 & 0 & 13 \\
\hline La Razón & 14 & 0 & 1 & 9 \\
\hline El Español & 3 & 2 & 1 & 8 \\
\hline El Mundo & 14 & 6 & 2 & 21 \\
\hline Eldiario.es & 15 & 2 & 0 & 17 \\
\hline Okdiario & 8 & 0 & 0 & 14 \\
\hline $\begin{array}{l}\text { El Economista } \\
\text { digital }\end{array}$ & 3 & 2 & 0 & 3 \\
\hline El País & 4 & 0 & 0 & 0 \\
\hline Huffpost & 1 & 0 & 0 & 0 \\
\hline
\end{tabular}

Fuente: MyNews

\section{Tercer desafío: Evitar la criminalización de los grupos sociales}

Por último, como tercer desafío se analizará algunos ejemplos en los cuales se responsabilizan a los jóvenes por la propagación del virus, especialmente se destaca hechos y casos donde se infringen las restricciones de los estados de emergencia y alarma. Este análisis se vincula al desafío de responsabilidad social de los medios informativos de no reforzar estereotipos y prejuicios.

En reportajes como “¿Por qué la gente se salta la cuarentena?” de la revista XLSemanal (Goitia, 2020), se asume como algo tradicional e ineludible el rol infractor de los jóvenes con la siguiente frase: “Ocurre, además en todas las edades. No sólo los jóvenes, aunque, según las encuestas, sean estos quienes menos amenazados se sienten por la pandemia". Lo anterior se comprueba en la inclusión del fragmento "no sólo los jóvenes". 
Las fiestas prohibidas han sido uno de los temas principales en la representación de los jóvenes como grupo infractor. El diario chileno La Tercera (Retamal, 2020) publicó, el 3 de mayo de 2020, la noticia "Sorprende a 400 personas en fiesta clandestina en camino a Melipilla durante el toque de queda". En el segundo párrafo se afirma que "un grupo de jóvenes respondió a una convocatoria viralizada en las redes sociales" marcándose con negrita. Cabe destacar que la fuente utilizada, la declaración de un policía chileno, no menciona una responsabilidad de un grupo específico. La responsabilidad a un grupo específico, no estando incluso confirmada, puede generar reacciones de rechazo en los receptores. Así se observó en la sección de comentarios donde se criticó a los jóvenes como carentes de inteligencia y cegados por el consumo de drogas. Es importante mencionar el enfoque específico de este texto que lo diferencia de otros que cubrieron el mismo hecho, pero no atribuyeron responsabilidad. En otro país sudamericano, como Perú, el diario Comercio (Redacción EC, 2020) publicó el 4 de abril otra noticia sobre la realización de fiestas prohibidas organizadas por jóvenes, con el título "Cañete: soldados intervienen a 30 jóvenes en fiesta y los obligan a trotar hacia base militar". A diferencia de la noticia de La Tercera, la responsabilización tiene mayor visibilidad al incorporarse en el titular. Otros ejemplos de la representación de los jóvenes en su rol infractor se añaden en el siguiente cuadro, los cuales han sido recopilados en Google noticias:

Tabla: 2: Ejemplo de noticias referente a multas a jóvenes en sus titulares

\begin{tabular}{|l|l|l|}
\hline \multicolumn{1}{|c|}{ Titular } & \multicolumn{1}{|c|}{$\begin{array}{c}\text { Fecha de } \\
\text { publicación }\end{array}$} & \multicolumn{1}{c|}{ Medio } \\
\hline $\begin{array}{l}\text { Multa de 600 euros a dos jóvenes por saltarse el estado } \\
\text { de alarma }\end{array}$ & 17 de marzo & $\begin{array}{l}\text { Ahora } \\
\text { Córdoba }\end{array}$ \\
\hline $\begin{array}{l}\text { Multitud de jóvenes multados en Leganés por hacer } \\
\text { una fiesta detectada por un helicóptero de la policía }\end{array}$ & 20 de marzo & Telemadrid \\
\hline $\begin{array}{l}\text { La policía local de Granada denuncia a 50 jóvenes por } \\
\text { fiestas en pisos }\end{array}$ & 15 de marzo & Ideal \\
\hline $\begin{array}{l}\text { Sancionados 7 jóvenes por hacer una fiesta en una zona } \\
\text { común de un edificio de General Dávila }\end{array}$ & 20 de abril & $\begin{array}{l}\text { El diario } \\
\text { Montañes }\end{array}$ \\
\hline
\end{tabular}

\section{Fuente: Google Noticias}

La culpabilización por infringir las normativas también ha sido generada desde el discurso político. Sobre este aspecto citamos dos ejemplos, donde los periodistas insertan declaraciones de políticos en las bajadas de las noticias. El primer texto del diario de Burgos (Ical, 2020) se titula "De la Rosa no acepta pasar de fase y confía en los expertos" y divulga las declaraciones del alcalde de esta ciudad española sobre las medidas para reducir las restricciones a la movilidad. La crítica hacia el comportamiento de los jóvenes no se expresa en el titular, pero sí en la bajada de la noticia con la siguiente frase: "El alcalde de Burgos denuncia la actitud de los jóvenes que se concentran en la calle a última hora de la tarde e insta a la Policía Local y Nacional a actuar para corregir estos "comportamientos irregulares"'”. En el cuerpo de la noticia, no será hasta el 5 párrafo cuando se retoma la crítica hacia el comportamiento de la juventud en la pandemia. Cabe destacar, que la información es apoyada por una imagen donde se retrata un grupo de jóvenes reunidos con sus perros. El segundo texto es titulado "Un menor ingresado en la 
UCI del Hospital General de Castellón con sospechas de Covid-19" del diario El Mundo (2020), donde se inserta la declaración de la consellera de Sanidad de la Comunidad Valenciana, Ana Barceló, de la siguiente forma: "La consellera de Sanidad Ana Barceló, pedía este jueves precisamente "responsabilidad" a los jóvenes: "Esto no va en serio, no es una película de ficción".

\section{Conclusiones}

La pandemia del Covid-19 pone en evidencia los problemas estructurales de un periodismo tradicional que aplica un modelo de bajo coste, donde prima la cantidad por sobre la calidad. De manera reciente, se puede observar este tipo de lógica en los despidos de periodistas y el cierre de delegaciones. La escasez de recursos aplicada por esta lógica empresarial es contraproducente con un contexto de emergencia, como es el caso de la pandemia del Covid-19, al necesitarse contenidos de mayor calidad, rigurosidad y profundización para entender la complejidad de este escenario. En este artículo hemos visto cómo algunos medios informativos españoles otorgan mayor relevancia al dato del número de muertos por sobre la cantidad de contagios, a pesar de que este último nos permite comprender la evolución de la pandemia y el grado de efectividad de las medidas de contención. Esto se explica por la tendencia de los medios informativos de centrarse en los efectos visibles de la violencia, definido como violencia directa, y dejar en segundo plano el análisis de las causas y los factores que la explican, conceptualizado como violencia estructural (Galtung, 1998). Por su parte, la reiteración de una visión catastrofista, expresada en el número de muertos y hechos trágicos, contribuye a que las personas experimenten el síndrome del mundo cruel, o la interpretación del contexto cercano como un ambiente peligroso, amenazador y negativo (Gerbner, 1998).

La transformación del periodismo, a un modelo más constructivo y pacífico, pasa por modificar los parámetros, valores noticiosos o criterios de noticiabilidad que rigen actualmente la profesión. De esta manera, es necesario reducir el peso de las malas noticias aportando contenidos sobre la denuncia de los problemas estructurales, de sus soluciones y la divulgación de noticias positivas. Esto también invita a reflexionar sobre los modelos de financiación y organización para poder plasmar estos cambios, en la búsqueda de la independencia periodística y de construir unas redacciones más horizontales.

Sobre la representación de los jóvenes, se ha visualizado ejemplos donde existe una criminalización hacia este grupo, en coherencia con su tradicional asociación como grupo infractor de las normativas y el bien común. En la cobertura de estos hechos, si son fiestas, es necesario tener la certeza de si efectivamente participó un grupo etario específico, como también conocer cuál es el rango de edad de la delimitación de juventud. Esto dado que puede ser un evento en el cual participan también adultos, y estaríamos con ello focalizando la responsabilidad en un grupo específico. El rango que se usa para clasificar a un niño, un joven y un adulto varía dependiendo el país. Al respecto, 
Krauskopf (2015) publica estos parámetros para los países latinoamericanos. Algunos como Chile el ser joven comprende de los 15 a los 29 años mientras que en Uruguay esta etapa empieza a los 14 años y se extiende a los 25 años. Los ejemplos vistos sobre la construcción de la juventud como grupo infractor nos permiten conocer diferentes estrategias, desde la inclusión de una declaración en el titular que atribuye la responsabilidad a la propia culpabilización por parte del medio informativo.

\section{Bibliografía}

Arévalo Salinas, A. (2020). Periodismo de paz y conflictos. Valencia: Tirant Humanidades.

Asociación Española de Comunicación Científica (2020). Consejos para informar sobre el nuevo coronavirus. Recuperado de: https://www.aecomunicacioncientifica.org/consejos-para-informar-sobre-elcoronavirus/

Asociación Diomira (2007). La imagen de los jóvenes en los medios de comunicación: ¿real y objetiva? Comunicación e juventude. En: Álvarez, L.; Evans, J.; Crespo, O. (Ed.), Comunicación e xuventude (pp. 309-316). Santiago de Compostela: Colexio Profesional de Xornalistas de Galicia

Arango-Kure, M., Garz, M. \& Rott, A. (2014). Bad news sells: The demand for news magazines and the tone of their covers. Journal of Media Economics, 27(4), 199214. doi:10.1080/08997764.2014.963230

Barranquero, A. (2020). El Periodismo Social como área de especialización, perspectiva de reforma y cultura profesional. Una revisión de conceptos y debates. Estudios sobre el Mensaje Periodístico, 25(2), pp. 657-676. https://doi.org/10.5209/esmp.64794

Casares, A. (2020). Periodismo constructivo en tiempos de coronavirus. Ethic, 18 de mayo. Recuperado de: https://ethic.es/2020/05/periodismo-constructivo-entiempos-de-coronavirus/

Chomshy, N. \& Herman, E (2009). Los guardianes de la libertad. Crítica: Barcelona

Diario El Mundo (8 de mayo de 2020). Un menor ingresado en la UCI del Hospital General de Castellón con sospechas de Covid-19 en el inicio de la desescalada. Diario El Mundo. Recuperado de: https://www.elmundo.es/comunidadvalenciana/castellon/2020/05/08/5eb4f31021efa0ca6b8b45c4.html

Díaz Rojo, J. A. (2009). La polémica, las "buenas noticias" y las "malas noticias" como valores noticiosos predominantes en el discurso sobre la salud en tres diarios nacionales españoles. Tonos, Revista electrónica de Estudios Filológicos, 17. 
Recuperado de https://www.um.es/tonosdigital/znum17/secciones/estudios-4malasnoticias.htm

Domínguez-Panamá, J. (2017). El periodismo de desastre: de las no-rutinas a las funciones sociales del periodista. Comhumanitas: revista científica de comunicación, 8(1), pp. 103-115.

Ente de Comunicación Nacional en Argentina Enacom (2020). Recomendaciones de ENACOM ante la emergencia sanitaria. Recuperado de: https://www.enacom.gob.ar/institucional/recomendaciones-frente-alcoronavirus_n2189

Goitia, F. (24 de marzo de 2020). ¿Por qué la gente se salta la cuarentena? El psicópata del piso del lado. Revista XLSemanal. Recuperado de: https://www.xlsemanal.com/conocer/sociedad/20200324/saltarse-la-cuarentenaegoismo-falta-de-empatia-coronavirus.html

Galtung, G. (1998). Tras la violencia, 3R: reconstrucción, reconciliación, resolución. Afrontando los efectos visiones e invisibles de la guerra y la violencia. Bilbao, España: Bakeas.

Gerbner, G. (1998). Cultivation analysis: An overview. Mass communication and society, l(3-4), pp. 175-194. Doi https://doi.org/10.1080/15205436.1998.9677855

Ical (6 de mayo de 2020). De la Rosa acepta no pasar de fase y confía en los expertos. Diario de Burgos. Recuperado de: https://www.diariodeburgos.es/noticia/Z307E4E4B-F43C-78AEFA464C9C2B46D69E/202005/De-la-Rosa-acepta-no-pasar-de-fase-y-confia-enlos-expertos

Krauskopf, D. (2015). Los marcadores de juventud: La complejidad de las edades. Ultima década, 23(42), pp. 115-128. $\quad$ https://dx.doi.org/10.4067/S0718$\underline{22362015000100006}$

Lamberson, P. J., \& Soroka, S. (2018). A model of attentiveness to outlying news. Journal of Communication, 68(5), pp. 942-964. doi:10.1093/joc/jqy040

Menéndez, T. (2014). La presentación de los jóvenes en los medios de comunicación. Prensa, radio y televisión. En: Centro Reina Sofía sobre Adolescencia y Juventud Fundación de Ayuda contra la Drogadicción (FAD) (Eds.), Jóvenes y medios de comunicación: El desafio de tener que entenderse (pp. 26-35). Madrid: Centro Reina Sofía.

Redacción EC (4 de mayo de 2020). Canete: soldados intervienen a 30 jóvenes en fiesta y los obligan a trotar hacia base militar. Diario El Comercio de Perú. Recuperado de: https://elcomercio.pe/peru/canete-soldados-intervienen-a-30-jovenes-enfiesta-y-los-obligan-a-trotar-hasta-base-militar-nnpp-noticia/ 
Retamal, F. (3 de mayo de 2020). Sorprenden a 400 personas en fiesta clandestina en camino a Melipilla durante el toque de queda. Diario La Tercera. Recuperado de: https://www.latercera.com/nacional/noticia/sorprenden-a-400-personas-enfiesta-clandestina-en-camino-a-melipilla-durante-el-toque-dequeda/JETDRO2KFFBWFCWZ3R6PWEMFTY/

Soroka, S., \& McAdams, S. (2015). News, politics, and negativity. Political Communication, 32(1), pp. 1-22. doi:10.1080/10584609.2014.881942

Wolton, D. (2002). War Game. Información y Guerra. Editorial Siglo XXI. México

\section{Notas}

\footnotetext{
${ }^{\mathrm{i}}$ Los valores noticiosos o criterios de noticiabilidad son una serie parámetros que se utilizan para definir la importancia de los hechos y temas en cuanto a su valor como texto informativo.
} 